\title{
Effects of a 6-month exercise programme on disease activity, physical and functional parameters in patients with ankylosing spondylitis: Randomised controlled trial
}

\begin{tabular}{|c|c|}
\hline \multicolumn{2}{|c|}{$\begin{array}{l}\text { Authors: } \\
\text { Kim Nolte }{ }^{1} \\
\text { Dina C. Janse van Rensburg }{ }^{2} \\
\text { Lizelle Fletcher }\end{array}$} \\
\hline \multicolumn{2}{|c|}{$\begin{array}{l}\text { Affiliations: } \\
{ }^{1} \text { Department of Physiology, } \\
\text { Division of Biokinetics and } \\
\text { Sport Science, Faculty of } \\
\text { Health Sciences, School } \\
\text { of Medicine, University of } \\
\text { Pretoria, Pretoria, } \\
\text { South Africa }\end{array}$} \\
\hline \multicolumn{2}{|c|}{$\begin{array}{l}{ }^{2} \text { Section Sport Medicine, } \\
\text { Faculty of Health Sciences, } \\
\text { University of Pretoria, } \\
\text { Pretoria, South Africa }\end{array}$} \\
\hline \multicolumn{2}{|c|}{$\begin{array}{l}{ }^{3} \text { Department of Statistics, } \\
\text { Faculty of Natural and } \\
\text { Agricultural Sciences, } \\
\text { University of Pretoria, } \\
\text { Pretoria, South Africa }\end{array}$} \\
\hline \multicolumn{2}{|c|}{$\begin{array}{l}\text { Corresponding author: } \\
\text { Kim Nolte, } \\
\text { kim.nolte@up.ac.za }\end{array}$} \\
\hline \multicolumn{2}{|c|}{$\begin{array}{l}\text { Dates: } \\
\text { Received: } 09 \text { Dec. } 2020 \\
\text { Accepted: } 19 \text { Mar. } 2021 \\
\text { Published: } 29 \text { June } 2021\end{array}$} \\
\hline \multicolumn{2}{|c|}{$\begin{array}{l}\text { How to cite this article: } \\
\text { Nolte, K., Janse van } \\
\text { Rensburg, D.C. \& Fletcher, L., } \\
2021 \text {, 'Effects of a 6-month } \\
\text { exercise programme on } \\
\text { disease activity, physical and } \\
\text { functional parameters in } \\
\text { patients with ankylosing } \\
\text { spondylitis: Randomised } \\
\text { controlled trial', South African } \\
\text { Journal of Physiotherapy } \\
\text { 77(1), a1546. https://doi. } \\
\text { org/10.4102/sajp.v77i1.1546 }\end{array}$} \\
\hline \multicolumn{2}{|l|}{ Read online: } \\
\hline 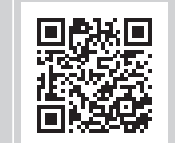 & $\begin{array}{l}\text { Scan this QR } \\
\text { code with your } \\
\text { smart phone or } \\
\text { mobile device } \\
\text { to read online. }\end{array}$ \\
\hline
\end{tabular}

Background: Exercise forms an important component in the management of ankylosing spondylitis (AS). The objective of our study was to determine the effects of a 6-month swimming, land-based stretching, strengthening and breathing exercise intervention in AS patients.

Methods/design: A total of 29 (14 females, 15 males) patients diagnosed with AS according to the Modified New York Criteria participated in our study. Participants were randomly assigned to an exercise group (ASE) (16 participants) and a control group (ASC) (13 participants). Erythrocyte sedimentation rate and C-reactive protein (CRP); anthropometric measurements; pulmonary function; aerobic capacity; balance; Bath AS Metrology Index; Bath AS Disease Activity Index and the Bath AS Functional Index were assessed.

Discussion: Erythrocyte sedimentation rate and CRP did not change notably within or between the groups from pre- to post-intervention. The between group analyses of the physical assessments favoured the ASE with observable improvements in chest expansion $(p=0.002)$, forced expiration volume $(p=0.012)$, absolute $(p=0.017)$ and relative $(p=0.003)$ maximal oxygen consumption, absolute $(p=0.028)$ and relative $(p=0.001)$ physical work capacity. Within the ASE, there is statistical evidence of improvements in 11 of the 19 physical variables. Within the ASC, five of the physical variables deteriorated substantially ( $p$ between 0.004 and 0.037 ) and only balance overall stability on the right improved $(p=0.016)$. The three functional assessments in the ASE improved $(p<0.007)$ compared with the ASC post-intervention. A 6-month combined exercise programme may improve physical and functional capacity of AS patients.

Conclusion: A multimodal exercise intervention may be useful in the management of ankylosing spondylitis.

Clinical implications: An unsupervised well -explained exercise programme combining swimming, land-based strengthening and stretching exercises and breathing exercises may be an option for patients with ankylosing spondylitis.

Trial registration: Registration not undertaken at the time of writing.

Keywords: ankylosing spondylitis; arthritis; aquatics; exercise therapy; rehabilitation.

\section{Introduction}

Ankylosing spondylitis (AS) is a chronic, systemic, rheumatic disease that is a prototype of seronegative spondyloarthropathies and characterised by inflammation, primarily of the spine (Ince, Sarpel \& Durgun 2006). The main clinical characteristics of AS are reduced physical activity, pain, stiffness, sleep disturbances, decreased spinal mobility and psychological consequences such as depression (Lim, Lim \& Lee 2005). Ankylosing spondylitis management strategies should focus on reducing disease activity, improving lumbar spinal mobility and functional status (Özdemir 2011). Treatment of AS usually includes the use of anti-inflammatory drugs to reduce pain and stiffness and disease modifying drugs to try to stop or prevent disease progression. Patients are also advised to exercise to maintain mobility of the spine and peripheral joints (Hidding et al. 1993). A growing body of research reveals that exercise is as crucial as drug treatment in the management of AS (Dougados \& Van der Heijde 2002; Heikkilä et al. 2000; Nolte \& Janse van Rensburg 2001). Functional limitations in early AS may be closely related to symptoms, whereas functional limitations in long-standing AS may be more closely related to cumulative factors leading to spinal fusion and long-term structural damage (Ward et al. 2005).

Copyright: (C) 2021. The Authors. Licensee: AOSIS. This work is licensed under the Creative Commons Attribution License. 
In AS, the main objectives of exercise therapy are prevention and delay of stiffness, pain control, postural re-education and improvement of functional and cardiorespiratory function (O'Dwyer, O'Shea \& Wilson 2014). Considering the numerous structures and functions that are influenced by the disease, exercise prescription for AS patients is not simple and should probably include various modalities and types of exercises. The knowledge of aquatic exercise in AS is still limited (Zão \& Canista 2017). However, it appears as if water exercise may have additive beneficial effect on pain, mobility, functionality and quality of life (Fernández et al. 2014; Karapolat et al. 2009).

Few randomised controlled studies have examined the effects of long duration (more than 3 months) exercise programmes specifically combining swimming and land-based stretching, strengthening and breathing exercises in AS patients. It has also been recommended that the quality of interventions in exercise trials for patients with AS can be improved and that future trials should focus on measuring and reporting physiological responses and adherence to exercise intervention (Daginfrud et al. 2011). A recent meta-analysis of randomised controlled trials on the effects of exercise programmes in AS patients concluded that further studies are needed to investigate the effects of specific exercise interventions on pain, quality of life, function and disease activity (Hu et al. 2020). Our study aimed to determine the effects of a 6-month swimming, land-based stretching, strengthening and breathing exercise intervention on disease activity, physicaland functional-parameters in patients with AS by comparing an exercise group (ASE) with a control group (ASC).

\section{Methods}

A total of 29 patients (14 females, 15 males) diagnosed with AS according to the Modified New York Criteria, between the ages of 18-70 years were recruited for our study. Eligible patients were referred by rheumatologists in private and public settings. Ankylosing spondylitis is a rare disease with the reported prevalence in Africa at 7.4 per 10000 (Dean et al. 2014). Only patients in close proximity to the testing facility could be recruited, and were eligible to participate if they could communicate in English, knew how to swim, were not currently participating in a structured exercise programme and were on stable medication for the last 3 months. Ankylosing spondylitis medication (dosage and type) was not changed or adjusted during the period they were involved in our study. In case of disease exacerbation, participants were allowed to use oral analgesics or topical anti-inflammatory ointments. Exclusion criteria were an inability or unwillingness to participate in an exercise programme and comorbidity of heart, lung, liver or kidney disease as confirmed by the rheumatologist. Participants completed and signed informed consent prior to initiation of our study. Allocation to groups was not concealed and participants were randomly divided into two groups by the trial coordinator during their baseline assessment using a random number table: an ASE (16 participants [nine females, seven males]) and an ASC (13 participants [seven females, six males]). The participants and biokineticist (responsible for the assessments and intervention) were not blinded to the group allocation.

\section{Assessments}

Participants were assessed three times during the course of our study ( 0 months, 3 months and 6 months). Assessments at the start $(0$ months $)$ and 6 months are reported on. Assessments included inflammatory blood markers, as well as physical and functional assessments. The inflammatory blood markers comprised of erythrocyte sedimentation rate (ESR) and C-reactive protein (CRP). The physical assessments included anthropometric measurements to determine chest wall expansion (CWE) and body composition. Anthropometric measurements included: body mass $(\mathrm{kg})$, stature $(\mathrm{cm})$, four skin folds (triceps, biceps, subscapular and suprailiac), two bony breadth measurements (humerus and femur) and four circumference measurements (biceps contracted, standing calf, maximum chest and minimum chest). Anthropometric measurements were taken according to the International Society for the Advancement of Kinanthropometry (ISAK) guidelines (Marfell-Jones \& Olds 2006). Body mass index (BMI), percentage body fat (PBF) and lean body mass (LBM) were determined. Pulmonary function was assessed using a portable spirometer (Spiroflow 2000, USA). Standardised procedures conforming to the Series 'American Thoracic Society (ATS) / European Respiratory Society (ERS) Task Force: Standardisation of Lung Function Testing, Number 2: Standardisation of Spirometry' were used (Miller et al. 2005). The forced vital capacity (FVC, mL), the forced expiration volume at the first second (FEV1, mL) and FEV1/ FVC (\%) were recorded. Aerobic capacity was assessed using an incremental bicycle ergometer test.The test was started with an initial load of $25 \mathrm{~W}$ at a cadence of $60-70$ revolutions per minute, with an increment increase of $25 \mathrm{~W}$ until exhaustion. Physical work capacity (PWC) was recorded ( $\mathrm{w}$ and $\mathrm{w} / \mathrm{kg}$ ) and the Astrand-Rhyming protocol was used to calculate the estimated maximal oxygen consumption $\left(\mathrm{VO}_{2 \max }\right)(\mathrm{mL} / \mathrm{kg} / \mathrm{min})$ of each participant. Balance was assessed on the Biodex balance SD ${ }^{\mathrm{TM}}$ (Biodex, United Kingdom). The athletic single leg stability test was used to determine unilateral balance. Three familiarisation trials were allowed. Overall stability (right and left) was recorded. Spinal flexibility was determined using the Bath Ankylosing Spondylitis Metrology Index (BASMI). The BASMI is composed of five measurements: cervical rotation, tragus-to-wall distance, lumbar lateral flexion, modified Schober's distance and intermalleolar distance (Lim et al. 2005). Each measurement indicates either 0 (mild disease involvement), 1 (moderate disease involvement) or 2 (severe disease involvement) points, resulting in a total BASMI score $0-10$. Physical assessments were completed by a qualified biokineticist with an ISAK level 1 accreditation.

Participants were required to complete two questionnaires in English; the Bath Ankylosing Spondylitis Disease Activity Index (BASDAI) and the Bath Ankylosing Spondylitis Functional Index (BASFI). The BASDAI is a 
self-administered questionnaire consisting of six questions relating to the five major symptoms of fatigue, spinal pain, joint pain or swelling, areas of localised tenderness and morning stiffness measured in terms of severity and duration. The overall BASDAI score ranges from zero to 10 , with higher scores indicating more severe symptoms (Lim et al. 2005). The BASFI consists of eight questions on daily activities and two additional questions that assess patients' ability to cope with everyday life. The mean of the 10 scales gives the BASFI score (0-10), with higher scores indicating more severe impairment (Karatepe et al. 2005). The BASMI, BASFI and BASDAI are validated instruments to assess spinal flexibility, disease activity and functional index in patients with AS and have been used in numerous studies (O’Dwyeret al. 2014).

\section{Intervention}

Participants in the ASC were instructed to continue with their current lifestyle and were educated on a suitable exercise programme at the conclusion of our study. Participants in the ASE each received an individual session with the biokineticist. She went through the exercise programme to ensure that they understood and could perform the exercises properly. They were instructed to exercise three times a week and were required to keep an exercise logbook in order to determine compliance with the programme. Participants were not supervised during their training but could use the pool and exercise facilities of the institution. The exercise difficulty was increased each month or 4-week period to ensure adequate progression. The swimming programme initially consisted of $10 \mathrm{~min}-12 \mathrm{~min}$ of swimming (participant could select swimming stroke of choice and could rest as required during the swimming training session). At the end of the 6-month period, participants were required to swim for $30 \mathrm{~min}$ as continuously as possible (preferably freestyle swimming stroke at a rate of perceived exertion of 13-15). In addition to the swimming, participants were required to complete landbased exercises that included postural correction exercises, strengthening exercises, stretches and pulmonary or breathing exercises. Participants were given an exercise therapy ball at the initiation of our study as some of the land-based exercises required the use of such a ball. Landbased exercises also increased in difficulty each month (4-week period). An example of the land-based exercises is presented (Table 1).

\section{Statistical analysis}

International Business Machines Corporation Statistical Package for the Social Sciences Statistics 19 was used for the statistical analysis. As a result of the small sample size, nonparametric Wilcoxon signed ranks tests, which are analogous to paired sample $t$-tests, were conducted, and the medians are reported. Mann-Whitney U non-parametric tests, analogous to independent $t$-tests for two samples, were used to compare the two groups before the onset of our study to determine whether any one group differs from the other with regard to any of the important covariates. Post-intervention Mann-Whitney U-tests were also performed to compare the two groups after the 6-month intervention. These tests were based on the available sample sizes at that time. All reported $p$-values are two-tailed. Effect sizes (ES) are reported for the between group comparisons on results that indicated substantial differences between the two groups $(p=0.05)$. Cohen (1992) suggested $r=0.1$ constitutes a small effect size, $r=0.3$ a moderate effect size and $r=0.5$ a large effect size.

\section{Ethical considerations}

Ethical approval for the study was obtained from the Postgraduate and Ethics Committee of the University of Pretoria (Faculty of Humanities) on 29 February 2012, no clearance numbers were assigned at the time.

\section{Results}

A total of 29 (14 females, 15 males) human leukocyte antigen-B27 positive patients who were diagnosed according to the Modified New York Criteria participated in our study. A description of the participants per group (ASE and ASC groups) is presented (Table 2). Large $p$-values (between 0.09 and 1) confirmed that it is unlikely that the two groups differ with respect to their demographic characteristics. Four participants in the ASE group and five in the ASC group did not complete the exercise programme. The final numbers were $n=12$ (six males and six females) for the ASE group and $n=8$ (three males and five females) in the ASC group. The ASE group had a mean age (years) of (mean \pm SD) $42 \pm 15$, range (21-66) and the ASC group (mean \pm SD) $42 \pm 11$, range (28-63). There were various reasons for some of participants not completing the exercise programme. For the ASC group reasons included: time constraints, difficulty travelling for the assessments and work commitments. For the ASE group reasons included: time constraints, difficulty travelling and the motivation to continue with the exercise programme over a relatively long period of time. Only the data of participants who completed the programme were included in the statistical analysis.

\section{Between group analyses}

The two groups were comparable concerning all blood, physical and functional assessments before the onset of the intervention (Table 3 - Table 5). The blood assessments including ESR $(p=0.562)$ and CRP $(p=0.35)$ did not show appreciable changes between the groups after the intervention (Table 3). The physical assessments favoured the ASE group with evidence of the incompatibility of the data with the null hypothesis of no improvements in CWE ( $p=0.002)$, FEV1\% $(p=0.012)$, absolute and relative $\mathrm{VO}_{2 \max }(p=0.017 ; p=0.003)$ as well as absolute and relative $\operatorname{PWC}(p=0.028 ; p=0.001)$ as presented (Table 4). The ES all indicate a large effect. Comparing the post-intervention functional assessments of 
TABLE 1: Land-based exercises.

\begin{tabular}{|c|c|c|c|c|c|c|c|}
\hline Exercise & Month 1 & Month 2 & Month 3 & Exercise & Month 4 & Month 5 & Month 6 \\
\hline \multicolumn{8}{|c|}{ Postural correction and strengthening exercises } \\
\hline $\begin{array}{l}\text { Opposite arm } \\
\text { and leg lift }\end{array}$ & $\begin{array}{l}\text { 10-15 reps } \\
\text { ( } R \text { \& } L) \\
1-2 \text { sets } \\
\text { Performed on } \\
\text { hands and feet }\end{array}$ & $\begin{array}{l}\text { 10-15 reps } \\
\text { (R \& L) } \\
2-3 \text { sets } \\
\text { Performed on } \\
\text { hands and feet }\end{array}$ & $\begin{array}{l}10-15 \text { reps } \\
\text { (R \& } L) \\
2-3 \text { sets } \\
\text { Performed on } \\
\text { exercise ball }\end{array}$ & $\begin{array}{l}\text { Opposite arm and } \\
\text { leg lift }\end{array}$ & $\begin{array}{l}10-15 \text { reps ( } R \text { \& L) } \\
1-2 \text { sets } \\
\text { Performed prone } \\
\text { on the floor }\end{array}$ & $\begin{array}{l}10-15 \text { reps }(R \& L) \\
2-3 \text { sets } \\
\text { Performed prone on } \\
\text { the floor }\end{array}$ & $\begin{array}{l}10-15 \text { reps }(R \& L) \\
2-3 \text { sets } \\
\text { Performed prone on } \\
\text { the floor } \\
\text { Dumbbells }\end{array}$ \\
\hline $\begin{array}{l}\text { Standing shoulder } \\
\text { rolls (backwards) } \\
\text { with or without } \\
\text { resistance }\end{array}$ & $\begin{array}{l}10-15 \text { reps } \\
1-2 \text { sets }\end{array}$ & $\begin{array}{l}10-15 \text { reps } \\
2-3 \text { sets }\end{array}$ & $\begin{array}{l}15-20 \text { reps } \\
2-3 \text { sets } \\
\text { Dumbbells }\end{array}$ & Reverse flies & $\begin{array}{l}10-15 \text { reps } \\
1-2 \text { sets } \\
\text { Performed } \\
\text { standing }\end{array}$ & $\begin{array}{l}10-15 \text { reps } \\
2-3 \text { sets } \\
\text { Performed on exercise } \\
\text { ball }\end{array}$ & $\begin{array}{l}10-15 \text { reps } \\
2-3 \text { sets } \\
\text { Performed on exercise ball } \\
\text { Dumbbells }\end{array}$ \\
\hline $\begin{array}{l}\text { Hip extension } \\
\text { (hip lift) }\end{array}$ & $\begin{array}{l}15-20 \text { reps } \\
1-2 \text { sets }\end{array}$ & $\begin{array}{l}15-20 \text { reps } \\
2-3 \text { sets }\end{array}$ & $\begin{array}{l}10-15 \text { reps } \\
\text { (R \& L) } \\
2-3 \text { sets } \\
\text { Performed on } \\
\text { one leg }\end{array}$ & $\begin{array}{l}\text { Hip extension } \\
\text { (hip lift) }\end{array}$ & $\begin{array}{l}15-20 \text { reps } \\
2-3 \text { sets } \\
\text { Performed with } \\
\text { feet on exercise } \\
\text { ball }\end{array}$ & $\begin{array}{l}15-20 \text { reps } \\
3 \text { sets } \\
\text { Performed with feet } \\
\text { on exercise ball. Hold } \\
\text { last rep for } 20 \mathrm{~s}-30 \mathrm{~s}\end{array}$ & $\begin{array}{l}15-20 \text { reps } \\
R \& L \\
2-3 \text { sets } \\
\text { Performed with one foot } \\
\text { on exercise ball. Hold last } \\
\text { rep for } 10 \mathrm{~s}-15 \mathrm{~s}\end{array}$ \\
\hline Front plank & $\begin{array}{l}\text { Time held: } \\
30 \mathrm{~s}-60 \mathrm{~s} \\
1-2 \text { reps } \\
\text { Performed on } \\
\text { elbows and } \\
\text { feet }\end{array}$ & $\begin{array}{l}\text { Time held: } \\
30 \mathrm{~s}-60 \mathrm{~s} \\
2-3 \text { reps } \\
\text { Performed on } \\
\text { hands and feet }\end{array}$ & $\begin{array}{l}\text { Time held: } 60 \mathrm{~s} \\
2-3 \text { reps } \\
\text { Performed on } \\
\text { hands and feet }\end{array}$ & Front plank & $\begin{array}{l}\text { Time held: } \\
30 s-60 \mathrm{~s} \\
\mathrm{R} \& \mathrm{~L} \\
1-2 \text { reps } \\
\text { Performed with } \\
\text { one leg lifted }\end{array}$ & $\begin{array}{l}\text { Time held: } \\
30 s-60 \mathrm{~s} \\
\mathrm{R} \& \mathrm{~L} \\
2-3 \text { reps } \\
\text { Performed with one } \\
\text { leg lifted }\end{array}$ & $\begin{array}{l}\text { Time held: } \\
30 \mathrm{~s}-60 \mathrm{~s} \\
2-3 \text { reps } \\
\text { Performed with feet wide } \\
\text { and one arm across chest }\end{array}$ \\
\hline $\begin{array}{l}\text { Wall slides (with } \\
\text { exercise ball) }\end{array}$ & $\begin{array}{l}15-20 \text { reps } \\
1-2 \text { sets }\end{array}$ & $\begin{array}{l}20 \text { reps } \\
2-3 \text { sets }\end{array}$ & $\begin{array}{l}25-30 \text { reps } \\
3 \text { sets } \\
\text { Dumbbells }\end{array}$ & Lunges & $\begin{array}{l}20 \text { reps } \\
R \& L \\
2-3 \text { sets } \\
\text { Dumbbells } \\
\text { Static }\end{array}$ & $\begin{array}{l}20 \text { reps } \\
R \& L \\
2-3 \text { sets } \\
\text { Front lunge } \\
\text { Dumbbells }\end{array}$ & $\begin{array}{l}30 \text { reps } \\
R \& L \\
3 \text { sets } \\
\text { Walking lunge Dumbbells }\end{array}$ \\
\hline- & - & - & - & $\begin{array}{l}\text { Crunches on } \\
\text { exercise ball }\end{array}$ & $\begin{array}{l}12-15 \text { reps } \\
2 \text { sets }\end{array}$ & $\begin{array}{l}15 \text { reps } \\
2-3 \text { sets }\end{array}$ & $\begin{array}{l}10-12 \text { reps } \\
2-3 \text { sets } \\
\text { Reverse crunches } \\
\text { (feet on ball) }\end{array}$ \\
\hline \multicolumn{8}{|l|}{ Stretches } \\
\hline $\begin{array}{l}\text { Doorway chest } \\
\text { stretch }\end{array}$ & $\begin{array}{l}\text { Time held: } \\
20 \mathrm{~s} \\
\text { ( R \& L) } \\
2 \text { sets }\end{array}$ & $\begin{array}{l}\text { Time held: } \\
20 \mathrm{~s} \\
\text { (R \& L) } \\
\text { 3-4 sets }\end{array}$ & $\begin{array}{l}\text { Time held: } \\
30 \mathrm{~s} \\
\text { (R \& L) } \\
\text { 3-4 sets }\end{array}$ & $\begin{array}{l}\text { Doorway chest } \\
\text { stretch/side lunge } \\
\text { stretch }\end{array}$ & $\begin{array}{l}\text { Time held: } \\
20 \mathrm{~s} \\
\text { (R \& L) } \\
2 \text { sets }\end{array}$ & $\begin{array}{l}\text { Time held: } \\
20 \mathrm{~s} \\
\text { (R \& L) } \\
\text { 3-4 sets }\end{array}$ & $\begin{array}{l}\text { Time held: } \\
30 \mathrm{~s} \\
\text { (R \& L) } \\
\text { 3-4 sets }\end{array}$ \\
\hline Neck stretches & $\begin{array}{l}\text { Time held: } \\
10 \text { s each } \\
2 \text { sets }\end{array}$ & $\begin{array}{l}\text { Time held: } \\
10 \text { s each } \\
3-4 \text { sets }\end{array}$ & $\begin{array}{l}\text { Time held: } \\
10 \text { s each } \\
3-4 \text { sets }\end{array}$ & Neck stretches & $\begin{array}{l}\text { Time held: } \\
20 \text { s each } \\
3-4 \text { sets }\end{array}$ & $\begin{array}{l}\text { Time held: } \\
20 \text { s each } \\
3-4 \text { sets }\end{array}$ & $\begin{array}{l}\text { Time held: } \\
20 \text { s each } \\
3-4 \text { sets }\end{array}$ \\
\hline $\begin{array}{l}\text { Lying on back, } \\
\text { knees side to side } \\
\text { (low back) }\end{array}$ & $\begin{array}{l}15-20 \text { reps } \\
1-2 \text { sets }\end{array}$ & $\begin{array}{l}20 \text { reps } \\
1-2 \text { sets }\end{array}$ & $\begin{array}{l}25-30 \text { reps } \\
1-2 \text { sets }\end{array}$ & $\begin{array}{l}\text { Lying on back, knees } \\
\text { side to side (low back) } \\
\text { with feet off ground }\end{array}$ & $\begin{array}{l}10-12 \text { reps } \\
1-2 \text { sets }\end{array}$ & $\begin{array}{l}12-15 \text { reps } \\
2 \text { sets }\end{array}$ & $\begin{array}{l}12-15 \text { reps } \\
2-3 \text { sets }\end{array}$ \\
\hline $\begin{array}{l}\text { Hamstring stretch } \\
\text { with towel }\end{array}$ & $\begin{array}{l}\text { Time held: } \\
20 \mathrm{~s} \\
\text { (R \& L) } \\
2 \text { sets }\end{array}$ & $\begin{array}{l}\text { Time held: } \\
20 \text { s } \\
\text { (R \& L) } \\
\text { 3-4 sets }\end{array}$ & $\begin{array}{l}\text { Time held: } \\
30 \text { s } \\
\text { (R \& L) } \\
\text { 3-4 sets }\end{array}$ & $\begin{array}{l}\text { Hamstring stretch } \\
\text { with towel }\end{array}$ & $\begin{array}{l}\text { Time held: } \\
30 \mathrm{~s} \\
\text { (R \& L) } \\
\text { 3-4 sets }\end{array}$ & $\begin{array}{l}20 \text { reps } \\
\text { (R \& L) } \\
3 \text { sets } \\
\text { Dorsiflexion / } \\
\text { plantarflexion of ankle }\end{array}$ & $\begin{array}{l}30 \text { reps } \\
\text { (R \& L) } \\
3 \text { sets } \\
\text { Dorsiflexion / } \\
\text { plantarflexion of ankle }\end{array}$ \\
\hline $\begin{array}{l}\text { Abdominal and } \\
\text { chest breathing }\end{array}$ & $\begin{array}{l}10 \text { reps each } \\
1 \text { set }\end{array}$ & $\begin{array}{l}15 \text { reps each } \\
1 \text { set }\end{array}$ & $\begin{array}{l}15 \text { reps each } \\
2 \text { sets }\end{array}$ & $\begin{array}{l}\text { Bellows breathing, } \\
\text { diaphragmatic } \\
\text { breathing }\end{array}$ & $\begin{array}{l}15 s-30 s \\
2 \mathrm{~min}\end{array}$ & $\begin{array}{l}30 s-60 s \\
2 \mathrm{~min}\end{array}$ & $\begin{array}{l}30 s-60 s \\
2 \mathrm{~min}\end{array}$ \\
\hline $\begin{array}{l}\text { Lying on back } \\
\text { knee to chest } \\
\text { stretch (hip } \\
\text { flexors) }\end{array}$ & $\begin{array}{l}\text { Time held: } \\
20 \mathrm{~s} \\
\text { ( R \& L) } \\
2 \text { sets }\end{array}$ & $\begin{array}{l}\text { Time held: } \\
20 \text { s } \\
\text { (R \& L) } \\
\text { 3-4 sets }\end{array}$ & $\begin{array}{l}\text { Time held: } \\
30 \mathrm{~s} \\
\text { (R \& L) } \\
\text { 3-4 sets }\end{array}$ & $\begin{array}{l}\text { Lying on back knee } \\
\text { to chest stretch } \\
\text { (hip flexors) }\end{array}$ & $\begin{array}{l}\text { Time held: } \\
20 \mathrm{~s} \\
\text { (R \& L) } \\
2 \text { sets }\end{array}$ & $\begin{array}{l}\text { Time held: } \\
20 \mathrm{~s} \\
\text { (R \& L) } \\
2 \text { sets }\end{array}$ & $\begin{array}{l}\text { Time held: } \\
30 \mathrm{~s} \\
\text { (R \& L) } \\
2 \text { sets }\end{array}$ \\
\hline- & - & - & - & $\begin{array}{l}\text { Lying on back knee } \\
\text { to side (low back). } \\
\text { Opposite hand to } \\
\text { knee }\end{array}$ & $\begin{array}{l}\text { Time held: } \\
20 \mathrm{~s} \\
\text { (R \& L) } \\
2 \text { sets }\end{array}$ & $\begin{array}{l}\text { Time held: } \\
20 \mathrm{~s} \\
\text { (R \& L) } \\
2 \text { sets }\end{array}$ & $\begin{array}{l}\text { Time held: } \\
30 \mathrm{~s} \\
\text { (R \& L) } \\
2 \text { sets }\end{array}$ \\
\hline- & - & - & - & $\begin{array}{l}\text { Downward dog into } \\
\text { upward dog }\end{array}$ & $\begin{array}{l}\text { Time held: } \\
20 \mathrm{~s} \\
2 \text { sets }\end{array}$ & $\begin{array}{l}\text { Time held: } \\
20 \mathrm{~s} \\
2 \text { sets }\end{array}$ & $\begin{array}{l}\text { Time held: } \\
30 \mathrm{~s} \\
2 \text { sets }\end{array}$ \\
\hline
\end{tabular}

L, left; min, minutes; reps, repetitions; R, right; s, seconds.

TABLE 2: Description of the participants $(n=29)$.

\begin{tabular}{lcccccc}
\hline Variable & \multicolumn{2}{c}{ An exercise group } & & \multicolumn{2}{c}{ A control group } \\
\cline { 2 - 3 } \cline { 5 - 6 } & $\boldsymbol{n}=\mathbf{1 6}$ & Mean \pm SD & & $\boldsymbol{n}=\mathbf{1 3}$ & Mean \pm SD \\
\hline Age (years) & 39.44 & 14.8 & & 39.00 & 12.8 \\
Males $(n)$ & 9 & - & & 6 & - \\
Females $(n)$ & 7 & - & & 7 & - \\
Body mass $(\mathrm{kg})$ & 77.4 & 18.3 & & 81.1 & 21.5 \\
Stature $(\mathrm{cm})$ & 173.3 & 13.0 & & 174.2 & 8.8 \\
Body mass index $(\mathrm{BMI})$ & 25.7 & 5.5 & & 26.7 & 5.4 \\
\hline
\end{tabular}

the groups (Table 5) demonstrates that the ASE group improved in all variables, compared with the ASC group (BASFI, $p=0.007$; BASDAI, $p=0.002$; BASMI, $p=0.003$ ), with large ES.

\section{Within group analyses}

Within both groups, the inflammatory blood markers as measured by ESR and CRP did not change statistically ( $p$-ranges between 0.103 and 1, Table 3). The ASE group had statistical improvements in 11 of the 20 physical variables assessed as presented (Table 4). These included: PBF decreased $(p=0.001)$, LBM increased $(p=0.019)$, endomorphy decreased $(p=0.001)$, mesomorphy increased $(p=0.001)$, CWE increased $(p=0.008)$, absolute and relative $\mathrm{VO}_{2 \max }$ increased $(p=0.001 ; p=0.001)$, absolute and relative PWC increased ( $p=0.001 ; p=0.001)$ and balance overall stability (BOS) improved on the right ( $p=0.001$ ) and on the left $(p=0.014)$. In the ASC, there was statistical evidence of deterioration in five of the physical variables 
TABLE 3: Blood assessments pre-and post-intervention, within and between groups $(n=20)$.

\begin{tabular}{|c|c|c|c|c|c|c|c|c|c|c|}
\hline \multirow[t]{2}{*}{ Variable } & \multicolumn{4}{|c|}{$\begin{array}{l}\text { An exercise group ( } n=12) \\
\text { (Median) }\end{array}$} & \multicolumn{4}{|c|}{$\begin{array}{c}\text { A control group }(n=8) \\
\text { (Median) }\end{array}$} & \multicolumn{2}{|c|}{ Between group comparisons $(p)$} \\
\hline & Pre-intervention & Post-intervention & $p$ & $\uparrow / \downarrow$ & Pre-intervention & Post-intervention & $p$ & $\uparrow / \downarrow$ & Pre-intervention & Post-intervention \\
\hline ESR (mm/hr) & 2.00 & 2.00 & 0.707 & $=$ & 3.50 & 2.00 & 1.000 & $\downarrow$ & 0.936 & 0.562 \\
\hline $\mathrm{CRP}(\mu \mathrm{g} / \mathrm{mL})$ & 4.85 & 2.55 & 0.253 & $\downarrow$ & 3.00 & 6.00 & 0.103 & $\uparrow$ & 0.579 & 0.350 \\
\hline
\end{tabular}

CRP, C-reactive protein; ESR, erythrocyte sedimentation rate; $n$, post-intervention number.

TABLE 4: Physical assessments pre-and post-intervention, within and between groups $(n=20)$.

\begin{tabular}{|c|c|c|c|c|c|c|c|c|c|c|}
\hline \multirow[t]{2}{*}{ Variable } & \multicolumn{4}{|c|}{$\begin{array}{l}\text { An exercise group }(n=12) \\
\text { (Median) }\end{array}$} & \multicolumn{4}{|c|}{$\begin{array}{l}\text { A control group ( } n=8) \\
\text { (Median) }\end{array}$} & \multicolumn{2}{|c|}{$\begin{array}{c}\text { Between group } \\
\text { comparisons } p \text { (Effect size) }\end{array}$} \\
\hline & $\begin{array}{c}\text { Pre- } \\
\text { intervention }\end{array}$ & $\begin{array}{c}\text { Post- } \\
\text { intervention }\end{array}$ & $p$ & $\uparrow / \downarrow$ & $\begin{array}{c}\text { Pre- } \\
\text { intervention }\end{array}$ & $\begin{array}{l}\text { Post- } \\
\text { intervention }\end{array}$ & $p$ & $\uparrow / \downarrow$ & $\begin{array}{c}\text { Pre- } \\
\text { intervention }\end{array}$ & $\begin{array}{l}\text { Post- } \\
\text { intervention }\end{array}$ \\
\hline BM & 77.80 & 76.10 & 0.525 & $\downarrow$ & 75.10 & 74.05 & 0.472 & $\downarrow$ & 0.475 & 0.970 \\
\hline BMI & 25.60 & 24.70 & 0.597 & $\downarrow$ & 25.55 & 25.25 & 0.374 & $\downarrow$ & 0.503 & 0.792 \\
\hline PBF & 23.80 & 22.70 & $0.001 *$ & $\downarrow$ & 30.75 & 32.00 & 0.410 & $\uparrow$ & 0.092 & 0.098 \\
\hline LBM & 53.80 & 54.50 & $0.019 *$ & $\uparrow$ & 54.65 & 52.55 & 0.303 & $\downarrow$ & 0.880 & 0.624 \\
\hline Endomorphy & 5.50 & 4.45 & $0.001^{*}$ & $\downarrow$ & 5.80 & 6.00 & 0.733 & $\uparrow$ & 0.083 & 0.082 \\
\hline Mesomorphy & 4.90 & 5.30 & $0.001 *$ & $\uparrow$ & 4.50 & 4.40 & $0.004 *$ & $\downarrow$ & 0.424 & 0.471 \\
\hline Ectomorphy & 1.40 & 1.65 & 0.460 & $\uparrow$ & 1.25 & 1.20 & 0.367 & $\downarrow$ & 0.589 & 0.678 \\
\hline CWE & 3.00 & 5.15 & $0.008^{*}$ & $\uparrow$ & 4.05 & 3.10 & $0.023 *$ & $\downarrow$ & 0.423 & $\begin{array}{c}0.002 * \\
(0.66)^{* *}\end{array}$ \\
\hline FEV1 (mL) & 3.10 & 3.12 & 0.940 & $\uparrow$ & 2.93 & 2.97 & 0.673 & $\uparrow$ & 0.440 & 0.518 \\
\hline FEV1\% & 102.85 & 109.00 & 0.124 & $\uparrow$ & 88.10 & 79.00 & 0.173 & $\downarrow$ & 0.254 & $\begin{array}{c}0.012 * \\
(0.55)^{* *}\end{array}$ \\
\hline FEV1/FVC (\%) & 100.50 & 96.50 & 0.129 & $\downarrow$ & 94.00 & 91.00 & 0.673 & $\downarrow$ & 1.000 & 0.518 \\
\hline PEF (L/min) & 7.72 & 8.03 & 0.291 & $\uparrow$ & 7.44 & 7.28 & 1.000 & $\downarrow$ & 0.683 & 0.518 \\
\hline $\mathrm{VO}_{2 \max }$ absolute (L/min) & 2.45 & 3.55 & $0.001 *$ & $\uparrow$ & 2.60 & 2.30 & $0.024 *$ & $\downarrow$ & 0.486 & $\begin{array}{c}0.017 * \\
(0.53)^{* *}\end{array}$ \\
\hline $\mathrm{VO}_{2 \max }$ relative $(\mathrm{mL} / \mathrm{kg} / \mathrm{min})$ & 32.25 & 43.10 & $0.001 *$ & $\uparrow$ & 32.50 & 25.30 & 0.073 & $\downarrow$ & 0.249 & $\begin{array}{c}0.003 * \\
(0.64)^{* *}\end{array}$ \\
\hline PWC absolute (w) & 107.00 & 145.00 & $0.001 *$ & $\uparrow$ & 130.00 & 115.00 & $0.037 *$ & $\downarrow$ & 0.682 & $\begin{array}{c}0.028 * \\
(0.49)^{* *}\end{array}$ \\
\hline PWC relative (w/kg) & 1.40 & 1.90 & $0.001 *$ & $\uparrow$ & 1.30 & 1.10 & $0.004 *$ & $\downarrow$ & 0.475 & $\begin{array}{c}0.001 * \\
(0.68)^{* *}\end{array}$ \\
\hline BOS right & 2.45 & 1.75 & $0.001 *$ & $\downarrow$ & 3.10 & 3.00 & $0.016^{*}$ & $\downarrow$ & 0.170 & 0.082 \\
\hline BOS left & 2.95 & 1.70 & $0.014 *$ & $\downarrow$ & 2.30 & 2.50 & 0.964 & $\uparrow$ & 0.812 & 0.167 \\
\hline
\end{tabular}

TABLE 5: Functional assessments pre-and post-intervention, within and between groups $(n=20)$.

\begin{tabular}{|c|c|c|c|c|c|c|c|c|c|c|}
\hline \multirow[t]{2}{*}{ Variable } & \multicolumn{4}{|c|}{$\begin{array}{l}\text { An exercise group }(n=12) \\
\text { (Median) }\end{array}$} & \multicolumn{4}{|c|}{$\begin{array}{l}\text { A control group ( } n=8) \\
\text { (Median) }\end{array}$} & \multicolumn{2}{|c|}{$\begin{array}{l}\text { Between group comparisons } p \\
\text { (Effect size) }\end{array}$} \\
\hline & $\begin{array}{c}\text { Pre- } \\
\text { intervention }\end{array}$ & $\begin{array}{c}\text { Post- } \\
\text { intervention }\end{array}$ & $p$ & $\uparrow / \downarrow$ & $\begin{array}{c}\text { Pre- } \\
\text { intervention }\end{array}$ & $\begin{array}{c}\text { Post- } \\
\text { intervention }\end{array}$ & $p$ & $\uparrow / \downarrow$ & $\begin{array}{c}\text { Pre- } \\
\text { intervention }\end{array}$ & $\begin{array}{c}\text { Post- } \\
\text { intervention }\end{array}$ \\
\hline BASFI & 2.48 & 0.95 & 0.798 & $\downarrow$ & 4.18 & 4.7 & 0.848 & $\uparrow$ & 0.056 & $\begin{array}{c}0.007 * \\
(0.59)^{* *}\end{array}$ \\
\hline BASDAI & 4.04 & 1.56 & $0.032 *$ & $\downarrow$ & 5.89 & 5.15 & 1.000 & $\downarrow$ & 0.779 & $\begin{array}{c}0.002^{*} \\
(0.67)^{* *}\end{array}$ \\
\hline
\end{tabular}

BASDAI, bath ankylosing spondylitis disease index; BASFI, bath ankylosing spondylitis functional index; BASMI, bath ankylosing spondylitis metrology index; $n$, post-intervention number; $\uparrow / \downarrow$, direction of change.

$*, p<0.05$.

$* *$, Effect size.

assessed, including mesomorphy $(p=0.004)$, CWE $(p=0.023)$ absolute $\mathrm{VO}_{2 \max }(p=0.024)$ and absolute and relative PWC $(p=0.037 ; p=0.004)$ as shown (Table 4$)$. The ASC group showed improvement only in BOS on the right $(p=0.016)$. The functional assessment results (Table 5) for the ASE group post-intervention had evidence of improvements in the BASDAI $(p=0.032)$ and $\operatorname{BASMI}(p=0.001)$ whilst the ASC group remained unchanged.

\section{Discussion}

Our study is one of few that has been carried out over a 6-month period, and consisted of a combination of exercises (land, swimming and breathing exercises) and involved a comprehensive assessment that included pulmonary function and balance (stability). The main findings of the 
effect of a 6-month exercise intervention in AS patients in this randomised control trial include: (1) no disease exacerbation with improved subjective (BASFI, BASDAI) and objective (CRP, BASMI) clinical parameters, (2) improvement of physical function, (3) improved chest expansion, (4) increased aerobic capacity and (5) better postural stability.

The first important finding was that the exercise intervention did not exacerbate the disease activity of the AS participants when considering the ESR and CRP. There was a clinical improvement within the ASE group's CRP value; however, the improvement was not statistically significant. Furthermore, the ASE group compared with the ASC group had better quality of life, fewer symptoms and improved spinal flexibility, confirming results from other studies (Dundar et al. 2014). Therefore, it appears as if the combined programme of swimming and land-based exercises can be performed without the fear of worsening disease activity and even lead to reduced disease activity and better quality of life as was shown in a previous study by our group where exercise leads to disease remission in rheumatoid arthritis (RA) patients (Janse van Rensburg et al. 2012).

The second important finding is that there was improvement in 11 of the physical assessment scores within the ASE group. The exercise intervention appears to have had a positive effect on the AS participants' anthropometric measurements and body composition. The ASE group had improvements in PBF, LBM and the endomorphy and ectomorphy components. This positive effect is very important as many AS patients tend to be sedentary and studies have shown that AS is associated with increased cardiovascular morbidity (Haroon et al. 2015). Therefore, the benefits of exercise such as improved body composition can possibly prevent or assist in the treatment of comorbid chronic conditions. Ankylosing spondylitis patients may have diseases such as Type II diabetes or hypertension. The general trend of the anthropometry and body composition scores within the ASC group was that of deterioration; however, statistically there were no differences between the ASE and ASC groups.

It has been shown that pulmonary involvement is common in patients with AS (Sampaio-Barros et al. 2007). Rigidity of the thorax occurs in AS with bony ankyloses of the thoracic vertebrae, costovertebral, costotransverse, sternoclavicular and sternomanubrial joints (Fisher, Cawley \& Holgate 1990). The third important finding of our 6-month exercise intervention is that this combination of exercises appears to be effective in improving thoracic expansibility in AS patients. The CWE improved within the ASE group whilst there was a deterioration within the ASC group. As a result, the CWE was statistically different between the two groups postintervention. The role of respiratory exercises in AS has been supported by previous studies (Daginfrud et al. 2011; Soloman et al. 1975; Drăgoi et al. 2016; So et al. 2012). Pulmonary function tests in AS have revealed a high prevalence of restrictive defects, characterised by a low FVC (SampaioBarros et al. 2007). The general trend of the pulmonary function values within the ASE group was that of small improvements. The results were inconsistent for the ASC group, all the pulmonary function values within this group decreased slightly except for FVC. The FEV1\% was statistically higher in the ASE group compared with the ASC group postintervention. Studies evaluating the effects of exercise on pulmonary functions are limited. Durmuset al. (2009) found statistically significant improvements in chest expansion and pulmonary function parameters such as FVC and FEV1 in AS patients following home-based exercises (Durmus et al. 2009). Ince et al. (2006) found a 3-month exercise intervention (multimodal exercise programme) also significantly increased chest expansion and FVC in AS patients (Ince et al. 2006). The results of our study are similar to those of Josenhans et al. (1971), who studied the effects of physiotherapy in 222 AS patients and found that pulmonary function parameters remained mostly unchanged despite improvements in chest wall and spinal mobility (Josenhans et al. 1971).

Aerobic exercises such as swimming or walking are often recommended in addition to conventional exercises for patients with AS (Elyan \& Khan 2008). A decrease in functional capacity can often be observed in patients with AS because of musculoskeletal or pulmonary impairment (Fisher et al. 1990). It has also been shown that there is an inverse relationship between cardiorespiratory fitness measured from an exercise test and the risk of mortality (Arena et al. 2007). In addition, recent studies have revealed that AS is also associated with an increased risk of cardiovascular morbidity (Haroon et al. 2015). Therefore, it is important that exercise interventions for patients with AS target other aspects of physical fitness (Dagfinrud et al. 2011). Considering the importance of aerobic capacity, the positive influence of the 6-month exercise programme on the $\mathrm{VO}_{2 \max }$ and $\mathrm{PWC}$ as the fourth finding is promising and emphasises the importance of exercising for patients with AS. Especially, as there was a substantial deterioration in the ASC group in their absolute $\mathrm{VO}_{2 \max }$ and PWC (absolute and relative) over a relatively short period of time and that the ASE group's aerobic capacity measures were higher compared with the ASC group at the end of our study. Other studies have shown similar improvements in aerobic capacity (Sveaas et al. 2014).

The postural changes characteristic of AS can result in changes in gait and balance impairment. These changes could lead to increased risk of falling (Zão \& Cantista 2017). Limited studies have evaluated the effect of specific programmes on strength, cardiorespiratory and functional factors such as balance (Millner et al. 2016). The fifth finding of our study was that the ASE group had a notable improvement in overall stability (right and left) as measured by the Biodex balance $\mathrm{SD}^{\mathrm{TM}}$. Although the ASC group had similar findings in overall stability on the right side, this may be because of repetition as a result of the testing that could have led to improvements. Parraca et al. (2011) found the Biodex balance $\mathrm{SD}^{\mathrm{TM}}$ to be a reliable and a useful measure of balance and risk of falls. Therefore, further research regarding the effect of exercise interventions on balance in AS patients is required (Parraca et al. 2011). 
Poor compliance and incorrect understanding on how to perform exercises are possible explanations of why exercise programmes fail. Also, the evidence supports the findings that supervised group exercise programmes are more effective than home exercise programmes (Cagliyan et al. 2007). However, studies comparing supervised- and homeexercise programmes are limited. In our study, participants in the exercise group each received an individual session with a biokineticist who went through the exercise programme to ensure that the participant understood and could perform the exercises properly. This could have contributed to the effectiveness of the exercise intervention. Furthermore, the ASE group kept an exercise log to determine compliance with the exercise programme. Compliance was acceptable as the AS participants attended $66.85 \%$ of their training sessions. Our study therefore supports the use of unsupervised programmes if supervision is not possible.

The limitations of our study include the small sample size of the two groups and the high drop-out rate. Larger samples will have more statistical power and allow parametric tests, which are more sensitive to detect changes, should there be any. Group allocation was not blinded for the assessor with a potential risk of bias. In addition, the same biokineticist who did the assessments conducted the intervention, which also may have biased the results. The wide age range and the groups containing both males and females could have also negatively influenced the results.

\section{Conclusions}

The main findings of our study show that the exercise intervention was effective in improving clinical parameters and physical and functional capacity without exacerbating the disease status of the participants. Therefore, it appears as if an unsupervised, well explained exercise programme combining swimming exercises, land-based strengthening and stretching exercises and breathing exercises may be a good option for patients with AS. These findings must, however, be considered within the light of the limitations and therefore should be interpreted with caution.

\section{Acknowledgements Competing interests}

The authors declare that they have no financial or personal relationships that may have inappropriately influenced them in writing this article.

\section{Authors' contributions}

K.N. was responsible for the conceptualisation, preparation, organisation and data collection for the study and the preparation and editing of the article. D.C.J. was responsible for the conceptualisation and data collection for the study and the preparation and editing of the article. L.F. was responsible for the data analysis and editing the article.

\section{Funding information}

Funding was received from the University of Pretoria's Research Development Fund.

\section{Data availability}

The authors confirm that the data supporting the findings of this study are available within the article.

\section{Disclaimer}

The views and opinions expressed in this article are those of the authors and do not necessarily reflect the official policy or position of any affiliated agency of the authors.

\section{References}

Arena, R., Myers, J., Williams, M.A., Gulati, M., Kligfield, P., Balady, G.J. et al., 2007, 'Assessment of functional capacity in clinical and research settings: A scientific statement from the american heart association committee on exercise, rehabilitation, and prevention of the council on clinical cardiology and the council on cardiovascular nursing', Circulation 116(3), 329-343. https://doi.org/10.1161/ on cardiovascular nursing,
circulationaha.106.184461

Cagliyan, A., Kotevoglu, N., Onal, T. \& Tekkus, B.B.K., 2007, 'Does group exercise program add anything more to patients with ankylosing spondylitis?', Journal of Back \& Musculoskeletal Rehabilitation 20(2-3), 79-85. https://doi.org/10.3233/ BMR-2007-202-305

Cohen, J., 1992, 'A power primer', Psychological Bulletin 112(2), 155-159. https://doi. org/10.1037/0033-2909.112.1.155

Dagfinrud, H., Halvorsen, S., Vøllestad, N.K., Niedermann, K., Kvien, T.K. \& Hagen, K.B., 2011, 'Exercise programs in trials for patients with ankylosing spondylitis: Do they really have the potential for effectiveness?', Arthritis Care \& Research (Hoboken) 63(4), 597-603. https://doi.org/10.1002/acr.20415

Dean, L.E., MacDonald, A.G., Downham, C., Sturrock, R.D. \& Macforlane, GJ., 2014 'Global prevalence of Ankylosing Spondylitis', Rhematology 53(4), 650-657. https://doi.org/10.1093/rheumatology/ket387

Dougados, M. \& Van der Heijde, D., 2002, 'Ankylosing spondylitis: How should the disease be assessed?', Best Practice \& Research: Clinical Rheumatology 16(4), 605-618. https://doi.org/10.1053/berh.2002.0252

Drăgoi, R.G., Amaricai, E., Drăgoi, M., Popoviciu, H. \& Avram, C., 2016, 'Inspiratory muscle training improves aerobic capacity and pulmonary function in patients with ankylosing spondylitis: A randomised controlled study', Clinical Rehabilitation 30(4), 340-346. https://doi.org/10.1177/0269215515578292

Dundar, U., Solak, O., Toktas, H., Demirdal, U.S., Subasi, V., Kavuncu, V. et al., 2014, 'Effect of aquatic exercise on ankylosing spondylitis: A randomised controlled trial', Rheumatology International 34(11), 1505-1511. https://doi.org/10.1007/ s00296-014-2980-8

Durmuş, D., Alayli, G., Uzun, O., Tander, B., Cantürk, F., Bek, Y. et al., 2009, 'Effects of two exercise interventions on pulmonary functions in the patients with ankylosing spondylitis', Joint Bone Spine 76(2), 150-155. https://doi.org/ 10.1016/j.jbspin.2008.06.013

Elyan, M. \& Khan, M.A., 2008, 'Does physical therapy still have a place in the treatment of ankylosing spondylitis?', Current Opinion in Rheumatology 20(3), 282-286. https://doi.org/10.1097/BOR.0b013e3282fa13c9

Fernández García, R., Sánchez-Sánchez, L.de.C., López Rodríguez, M.de.M. \& Sánchez Granados, G., 2015, 'Effects of an exercise and relaxation aquatic program in patients with spondyloarthritis: A randomised trial', Medicina Clinica (Barc) 145(9), 380-384. https://doi.org/10.1016/j.medcli.2014.10.015

Fisher, L.R., Cawley, M.I. \& Holgate, S.T., 1990, 'Relation between chest expansion, pulmonary function, and exercise tolerance in patients with ankylosing spondylitis', Annals of the Rheumatic Diseases 49(11), 921-925. https://doi. org/10.1136/ard.49.11.921

Haroon, N.N., Paterson, J.M., Li, P., Inman, R.D. \& Haroon, N., 2015, 'Patients with ankylosing spondylitis have increased cardiovascular and cerebrovascular mortality: A population-based study', Annuals of Internal Medicine 163(6), 409-416. https://doi.org/10.7326/m14-2470

Heikkilä, S., Viitanen, J.V., Kautiainen, H. \& Kauppi, M., 2000, 'Sensitivity to change of mobility tests: Effect of short term intensive physiotherapy and exercise on spinal, hip, and shoulder measurements in spondyloarthropathy', Journal of Rheumatology 27(5), 1251-1256.

Hidding, A., Van der Linden, S., Boers, M., Gielen, X., De Witte, L., Kester, A. et al., 1993 , 'Is group physical therapy superior to individualized therapy in ankylosing spondylitis? A randomised controlled trial', Arthritis Care \& Research 6(3), 117-125. https://doi.org/10.1002/art.1790060303

Hu, X.M., Chen, J.L., Tang, W.J., Chen, W.J., Sang, Y. \& Jia, L.N., 2020, 'Effects of exercise programmes on pain, disease activity and function in ankylosing spondylitis: A meta-analysis of randomised controlled trials', European Journal of Clinical Investigation 50(12), 1-10. https://doi.org/10.1111/eci.13352 
Ince, G., Sarpel, T. \& Durgun, B.S.E., 2006, 'Effects of a multimodal exercise program for people with ankylosing spondylitis', Physical Therapy 86(7), 924-935. https:// doi.org/10.1093/ptj/86.7.924

Janse van Rensburg, D.C., Ker, J.A., Grant, C.C. \& Fletcher, L., 2012, 'Effect of exercise on cardiac autonomic function in females with rheumatoid arthritis', Clinical Rheumatology 31(8), 1155-1162. https://doi.org/10.1007/s10067012-1985-5

Josenhans, W.T., Wang, C.S., Josenhans, G. \& Woodbury, J.F., 1971, 'Diaphragmatic contribution to ventilation in patients with ankylosing spondylitis', Respiration 28(4), 331-346. https://doi.org/10.1159/000192822

Karapolat, H., Eyigor, S., Zoghi, M., Akkoc, Y., Kirazli, Y. \& Keser, G., 2009, 'Are swimming or aerobic exercise better than conventional exercise in ankylosing spondylitis patients? A randomised controlled study', European Journal of Physical Rehabilitation Medicine 45(4), 449-457.

Karatepe, A.G., Akkoc, Y., Akar, S., Kirazli, Y. \& Akkoc, N., 2005, 'The Turkish versions of the bath ankylosing spondylitis and dougados functional indices: Reliability and validity', Rheumatology International 25(8), 612-618. https://doi.org/10.1007/ s00296-004-0481-x

Lim, H.J., Lim, H.S. \& Lee, M.S., 2005, 'Relationship between self-efficacy and exercise duration in patients with ankylosing spondylitis', Clinical Rheumatology 24(4), 442-443. https://doi.org/10.1007/s10067-004-0974-8

Marfell-Jones, M. \& Olds, T., 2006, International standards for anthropometric assessment, The International Society for Advancement in Kinanthropometry, Canberra.

Miller, M.R., Hankinson, V., Brusasco, V., Burgos, F, Casaburi, R., Coates, A. et al 2005, 'Series: "ATS/ERS task force: Standardisation of lung function testing"', European Respiratory Journal 26, 319-338. https://doi.org/10.1183/0903136.05. 00034805

Millner, J.R., Barron, J.S., Beinke, K.M., Butterworth, R.H., Chasle, B.E., Dutton, L.J. et al., 2016, 'Exercise for ankylosing spondylitis: An evidence-based consensus statement', Seminars Arthritis and Rheumatism 45(4), 411-427. https://doi. org/10.1016/j.semarthrit.2015.08.003
Nolte, K. \& Janse van Rensburg, D.C., 2001, 'The role of exercise in the rehabilitation of ankylosing spondylitis', International Sports Medicine Journal 2(4), 1-12.

O'Dwyer, T., O'Shea, F. \& Wilson, F.,2014, 'Exercise therapy for spondyloarthritis: A systematic review', Rheumatology International 34(7), 887-902. https://doi. org/10.1007/s00296-014-2965-7

Özdemir, O., 2011, 'Quality of life in patients with ankylosing spondylitis: Relationships with spinal mobility, disease activity and functional status', Rheumatology International 31(5), 605-660. https://doi.org/10.1007/s00296-009-1328-2

Parraca, J.A., Olivares, P.R., Carbonell-Baeza, A., Aparicio, V.A., Adsuar, J.C. \& Gusi, N., 2011,'Test-retest reliability of Biodex balance sd on physically active old people' Journal of Human Sport \& Exercise 6(2), 444-451. https://doi.org/10.4100/ jhse.2011.62.25

Sampaio-Barros, P.D., Cerqueira, E.M., Rezende, S.M., Maeda, L., Conde, R.A., Zanardi, V.A. et al., 2007 'Pulmonary involvement in ankylosing spondylitis', Clinical Rheumatology 26(2), 225-230. https://doi.org/10.1007/s10067-006-0286-2

So, M.W., Heo, H.M., Koo, B.S., Kim, Y.G., Lee, C.K. \& Yoo, B., 2012, 'Efficacy of incentive spirometer exercise on pulmonary functions of patients with ankylosing spondylitis stabilized by tumor necrosis factor inhibitor therapy', Journal of Rheumatology 39(9), 1854-1858. https://doi.org/10.3899/jrheum.120137

Soloman, L., Beighton, P., Valkenburg, H.A., Robin, G. \& Soskoline, C.L., 1975 'Rheumatic disorders in the South African Negro: Part 1: Rheumatoid Arthritis and Ankylosing Spondylitis', SA Medical Journal 49, 1292-1296.

Sveaas, S.H., Berg, I.J., Provan, S.A., Semb, A.G., Hagen, K.B., Vøllestad, N. et al., 2014 'Efficacy of high intensity exercise on disease activity and cardiovascular risk in active axial spondyloarthritis: A randomised controlled pilot study', PLoS One 9(9), e108688. https://doi.org/10.1371/journal.pone.0108688

Ward, M.M., Weisman, M.H., Davis, J.C. \& Reveille, Jr. J.D., 2005, 'Risk factors for functional limitations in patients with long-standing ankylosing spondylitis', Arthritis \& Rheumatology 53(5), 710-717. https://doi.org/10.1002/art.21444

Zão, A. \& Cantista, P., 2017, 'The role of land and aquatic exercise in ankylosing spondylitis: A systematic review', Rheumatology International 37(12), 1979-1990. https://doi.org/10.1007/s00296-017-3829-8 